\title{
Theory and Implementation of a Two-Step Unconditionally Stable Explicit Integration Algorithm for Vibration Analysis of Structures
}

\author{
Li Changqing, ${ }^{1}$ Jie Junping, ${ }^{1}$ Jiang Lizhong, ${ }^{1,2}$ and T. Y. Yang ${ }^{3,4}$ \\ ${ }^{1}$ School of Civil Engineering, Central South University, Changsha, Hunan 410075, China \\ ${ }^{2}$ National Engineering Laboratory for High Speed Railway Construction, Changsha 410075, China \\ ${ }^{3}$ International Joint Research Laboratory of Earthquake Engineering, Tongji University, 1239 Siping Road, Shanghai 2000092, China \\ ${ }^{4}$ Department of Civil Engineering, The University of British Columbia, 2329 West Mall, Vancouver, BC, Canada V6T 1Z4
}

Correspondence should be addressed to Li Changqing; lcq_stu@126.com

Received 29 June 2016; Revised 2 October 2016; Accepted 1 November 2016

Academic Editor: Mahmoud Bayat

Copyright (C) 2016 Li Changqing et al. This is an open access article distributed under the Creative Commons Attribution License, which permits unrestricted use, distribution, and reproduction in any medium, provided the original work is properly cited.

\begin{abstract}
Time history analysis is becoming the routine process to quantify the response of the structure under dynamic loads. In this paper, a novel two-step unconditionally stable explicit integration algorithm, named Unconditional Stable Two-Step Explicit Displacement Method (USTEDM), is proposed for vibration analysis of structure. USTEDM is unconditionally stable, requires low memory, produces no overshoot, and is third order accurate. The accuracy and efficiency of USTEDM are presented and compared with other commonly used integration algorithms. The result shows that the proposed algorithm has superior performance and can be used efficiently in solving vibration response of civil engineering structure.
\end{abstract}

\section{Introduction}

With the advance of computer hardware, more advanced computer software is being used to solve the response of the structure under dynamic loads. Hence, more advanced numerical methods have been developed to efficiently and accurately predict the dynamic response of the structure. In the past, multiple implicit [1-7] and explicit [8-12] integration algorithms have been developed, whose relationship [13] and design methods are comprehensively analyzed [14-16]. The explicit method has high computational efficiency, while the implicit method has higher stable. The commonly referenced explicit algorithm is Central Difference Method (CDM) (ref), $\mathrm{CDM}$ is easy to use, but it is only conditionally stable. Many new explicit methods which are unconditionally stable [17-19] have been developed. Though these methods are unconditionally stable, they require multiple steps which are not as efficient as the CDM. The paper presents a novel 2-step explicit integration method, named Unconditional Stable Two-Step Explicit Displacement Method (USTEDM), that is used to solve the dynamic response of a structure. USTEDM is efficient to use and unconditionally stable. The performance of USTEDM is compared with other explicit algorithms. The result shows that USTEDM has superior performance and can be used efficiently in solving vibration response of civil engineering structures.

\section{Derivation of the Two-Step Integration Algorithm}

Equation (1) shows the generalized dynamic equations of motion of a structure:

$$
m a(t)+c v(t)+k u(t)=f(t),
$$

where $a(t), v(t), u(t)$ are the acceleration, velocity, and displacement of the structure, respectively. $m, c, k$ are the mass, damping, and stiffness of the structure, respectively. $f(t)$ is external applied force. 
In this paper, a two-step integration algorithm, as presented in (2), is proposed to solve the dynamic equation as presented in (1):

$$
u_{t+2 \Delta t}=f\left(u_{t+\Delta t}, u_{t}, f_{t+2 \Delta t}, f_{t+\Delta t}, f_{t}, m, c, k, \Delta t\right),
$$

where $u_{t+2 \Delta t}$ is the displacement at time step $t+2 \Delta t$ and $\Delta t$ is the integration time step. Similarly, $u_{t+\Delta t}$ and $u_{t}$ are the displacement at time $t+\Delta t$ and $t$, while $f_{t+2 \Delta t}, f_{t+\Delta t}$ and $f_{t}$ are the force at $t+2 \Delta t, t+\Delta t$, and $t$, respectively.

It should be noted that (2) as presented above has physical units, where the displacement could be in the unit of "meter" or "feet," while the mass can be in the unit of "kg" or "slugs." To make the equation dimensionless, (2) can be rewritten in form of dimensionless quantity as

$$
\begin{aligned}
& \frac{u_{t+2 \Delta t}}{u_{t+\Delta t}}=\frac{u_{t+2 \Delta t}}{u_{t+\Delta t}}\left(1, \frac{u_{t}}{u_{t+\Delta t}}, \frac{f_{t+2 \Delta t} \Delta t^{2}}{m u_{t+\Delta t}}, \frac{f_{t+\Delta t} \Delta t^{2}}{m u_{t+\Delta t}}, \frac{f_{t} \Delta t^{2}}{m u_{t+\Delta t}},\right. \\
& \left.1, \frac{c \Delta t}{m}, \frac{k \Delta t^{2}}{m}, 1\right) .
\end{aligned}
$$

To construct function $u_{t+2 \Delta t} / u_{t+\Delta t}$ at the right hand of (3), the linear combination of $u_{t+2 \Delta t} / u_{t+\Delta t}$ with the arguments including 1, $\left(u_{t} / u_{t+\Delta t}\right)\left(f_{t+2 \Delta t} \Delta t^{2} / m u_{t+\Delta t}\right), f_{t+\Delta t} \Delta t^{2} / m u_{t+\Delta t}$ and $f_{t} \Delta t^{2} / m u_{t+\Delta t}$, can be used:

$$
\begin{aligned}
r_{1} \frac{u_{t+2 \Delta t}}{u_{t+\Delta t}}= & r_{2}+r_{3} \frac{u_{t}}{u_{t+\Delta t}}+r_{4} \frac{f_{t+2 \Delta t} \Delta t^{2}}{m u_{t+\Delta t}}+r_{5} \frac{f_{t+\Delta t} \Delta t^{2}}{m u_{t+\Delta t}} \\
& +r_{6} \frac{f_{t} \Delta t^{2}}{m u_{t+\Delta t}},
\end{aligned}
$$

where $r_{i}(i=1, \ldots, 6)$ are undetermined coefficients. The effects of the dimensionless arguments $c \Delta t / m, k \Delta t^{2} / m$ that represent the structure's material properties to the displacements can be expressed by replacing the coefficients $r_{i}(i=1,2,3)$ with linear combination of constant term $1, c \Delta t / m, k \Delta t^{2} / m$ :

$$
\begin{aligned}
& r_{1}=l_{1}+l_{2} \frac{c \Delta t}{m}+l_{3} \frac{k \Delta t^{2}}{m}, \\
& r_{2}=l_{4}+l_{5} \frac{c \Delta t}{m}+l_{6} \frac{k \Delta t^{2}}{m}, \\
& r_{3}=l_{7}+l_{8} \frac{c \Delta t}{m}+l_{9} \frac{k \Delta t^{2}}{m},
\end{aligned}
$$

where $l_{i}(i=1, \ldots, 9)$ are coefficients.

Substituting (5) into (4) yields

$$
\begin{aligned}
\left(l_{1}+\right. & \left.l_{2} \frac{c \Delta t}{m}+l_{3} \frac{k \Delta t^{2}}{m}\right) \frac{u_{t+2 \Delta t}}{u_{t+\Delta t}} \\
= & \left(l_{4}+l_{5} \frac{c \Delta t}{m}+l_{6} \frac{k \Delta t^{2}}{m}\right) \\
& +\left(l_{7}+l_{8} \frac{c \Delta t}{m}+l_{9} \frac{k \Delta t^{2}}{m}\right) \frac{u_{t}}{u_{t+\Delta t}}+l_{10} \frac{f_{t+2 \Delta t} \Delta t^{2}}{m u_{t+\Delta t}} \\
& +l_{11} \frac{f_{t+\Delta t} \Delta t^{2}}{m u_{t+\Delta t}}+l_{12} \frac{f_{t} \Delta t^{2}}{m u_{t+\Delta t}}
\end{aligned}
$$

where $l_{i}(i=1, \ldots, 12)$ are coefficients to be determined and $l_{10}=r_{4}, l_{11}=r_{5}, l_{12}=r_{6}$.

Equation (6) can be simplified to be

$$
\begin{aligned}
\left(l_{1} m\right. & \left.+l_{2} c \Delta t+l_{3} k \Delta t^{2}\right) u_{t+2 \Delta t} \\
= & \left(l_{4} m+l_{5} c \Delta t+l_{6} k \Delta t^{2}\right) u_{t+\Delta t} \\
& +\left(l_{7} m+l_{8} c \Delta t+l_{9} k \Delta t^{2}\right) u_{t}+l_{10} f_{t+2 \Delta t} \Delta t^{2} \\
& +l_{11} f_{t+\Delta t} \Delta t^{2}+l_{12} f_{t} \Delta t^{2} .
\end{aligned}
$$

In order to decrease the number of undetermined coefficients $l_{i}$, (7) can be simplified by dividing $l_{1}$ at both sides:

$$
\begin{aligned}
(m+ & \left.b_{1} c \Delta t+b_{2} k \Delta t^{2}\right) u_{t+2 \Delta t} \\
= & \left(b_{3} m+b_{4} c \Delta t+b_{5} k \Delta t^{2}\right) u_{t+\Delta t} \\
& +\left(b_{6} m+b_{7} c \Delta t+b_{8} k \Delta t^{2}\right) u_{t}+b_{9} f_{t+2 \Delta t} \Delta t^{2} \\
& +b_{10} f_{t+\Delta t} \Delta t^{2}+b_{11} f_{t} \Delta t^{2}
\end{aligned}
$$

where $b_{i}=\left(l_{i+1} / l_{1}\right)(i=1, \ldots, 11)$ are undetermined coefficients.

Equation (8) is the preliminary form of USTEDM.

\section{Determination of Coefficients}

3.1. Accuracy Analysis. The accuracy of USTEDM can be examined using convergence test. Equation (9) shows the Taylor series expansion of the displacement, velocity, and acceleration of the structure at time step of $t+2 \Delta t, t+\Delta t$, respectively.

$$
\begin{aligned}
& u_{(t+\Delta t)}=u_{(t)}+v_{(t)} d t+\frac{1}{2} a_{(t)} d t^{2}+\frac{1}{6} \dot{a}_{(t)} d t^{3}+\cdots, \\
& v_{(t+\Delta t)}=v_{(t)}+a_{(t)} d t+\frac{1}{2} \dot{a}_{(t)} d t^{2}+\frac{1}{6} \ddot{a}_{(t)} d t^{3}+\cdots, \\
& a_{(t+\Delta t)}=a_{(t)}+\dot{a}_{(t)} d t+\frac{1}{2} \ddot{a}_{(t)} d t^{2}+\frac{1}{6} \ddot{a}_{(t)} d t^{3}+\cdots, \\
& u_{(t+2 \Delta t)}=u_{(t)}+2 v_{(t)} d t+2 a_{(t)} d t^{2}+\frac{4}{3} \dot{a}_{(t)} d t^{3}+\cdots, \\
& v_{(t+2 \Delta t)}=v_{(t)}+2 a_{(t)} d t+2 \dot{a}_{(t)} d t^{2}+\frac{4}{3} \ddot{a}_{(t)} d t^{3}+\cdots, \\
& a_{(t+2 \Delta t)}=a_{(t)}+2 \dot{a}_{(t)} d t+2 \ddot{a}_{(t)} d t^{2}+\frac{4}{3} \ddot{a}_{(t)} d t^{3}+\cdots
\end{aligned}
$$

Similarly, the dynamic equilibriums at $t+2 \Delta t, t+\Delta t, t$ are expressed in

$$
\begin{aligned}
f_{t+2 \Delta t} & =m a_{(t+2 \Delta t)}+c v_{(t+2 \Delta t)}+k u_{(t+2 \Delta t)}, \\
f_{t+\Delta t} & =m a_{(t+\Delta t)}+c v_{(t+\Delta t)}+k u_{(t+\Delta t)}, \\
f_{t} & =m a_{(t)}+c v_{(t)}+k u_{(t)} .
\end{aligned}
$$


Substituting (9) into (10) yields

$$
\begin{aligned}
f_{t+2 \Delta t}= & m\left(a_{(t)}+2 \dot{a}_{(t)} d t+\cdots\right) \\
& +c\left(u_{(t)}+2 v_{(t)} d t+\cdots\right) \\
& +k\left(u_{(t)}+2 v_{(t)} d t+\cdots\right), \\
f_{t+\Delta t}= & m\left(a_{(t)}+\dot{a}_{(t)} d t+\cdots\right)+c\left(v_{(t)}+a_{(t)} d t+\cdots\right) \\
& +k\left(u_{(t)}+v_{(t)} d t+\cdots\right), \\
f_{t}= & m a_{(t)}+c v_{(t)}+k u_{(t)} .
\end{aligned}
$$

Substituting (9)-(11) into (8) yields

$$
\begin{aligned}
(m+ & \left.b_{1} c \Delta t+b_{2} k \Delta t^{2}\right) \varepsilon_{u_{t+2 \Delta t}} \\
= & \left(m+b_{1} c \Delta t+b_{2} k \Delta t^{2}\right) u_{(t+2 \Delta t)} \\
& -\left(b_{3} m+b_{4} c \Delta t+b_{5} k \Delta t^{2}\right) u_{(t+\Delta t)} \\
& -\left(b_{6} m+b_{7} c \Delta t+b_{8} k \Delta t^{2}\right) u_{(t)}-b_{9} f_{t+2 \Delta t} \Delta t^{2} \\
& -b_{10} f_{t+\Delta t} \Delta t^{2}-b_{11} f_{t} \Delta t^{2} \\
= & d_{0}+d_{1} \Delta t+d_{2} \Delta t^{2}+d_{3} \Delta t^{3}+\cdots,
\end{aligned}
$$

where

$$
\begin{aligned}
& d_{0}=\left(1-b_{3}-b_{6}\right) u_{(t)} m, \\
& d_{1}=\left(2-b_{3}\right) v_{(t)} m+\left(b_{1}-b_{4}-b_{7}\right) u_{(t)} c \text {, } \\
& d_{2}=\left(2-0.5 b_{3}-b_{9}-b_{10}-b_{11}\right) a_{(t)} m \\
& +\left(2 b_{1}-b_{4}-b_{9}-b_{10}-b_{11}\right) v_{(t)} c \\
& +\left(b_{2}-b_{5}-b_{8}-b_{9}-b_{10}-b_{11}\right) u_{(t)} k, \\
& d_{3}=\left(\frac{4}{3}-\frac{1}{6} b_{3}-2 b_{9}-b_{10}\right) \dot{a}_{(t)} m \\
& +\left(2 b_{1}-0.5 b_{4}-2 b_{9}-b_{10}\right) a_{(t)} c \\
& +\left(2 b_{2}-b_{5}-2 b_{9}-b_{10}\right) v_{(t)} k \text {, } \\
& d_{4}=\left(\frac{2}{3}-\frac{1}{24} b_{3}-2 b_{9}-\frac{1}{2} b_{10}\right) \ddot{a}_{(t)} m \\
& +\left(\frac{4}{3} b_{1}-\frac{1}{6} b_{4}-2 b_{9}-\frac{1}{2} b_{10}\right) \dot{a}_{(t)} c \\
& +\left(2 b_{2}-\frac{1}{2} b_{5}-2 b_{9}-\frac{1}{2} b_{10}\right) a_{(t)} k, \\
& d_{5}=\left(\frac{4}{15}-\frac{1}{120} b_{3}-\frac{4}{3} b_{9}-\frac{1}{6} b_{10}\right) \ddot{a}_{(t)} m \\
& +\left(\frac{2}{3} b_{1}-\frac{1}{24} b_{4}-\frac{4}{3} b_{9}-\frac{1}{6} b_{10}\right) \ddot{a}_{(t)} c \\
& +\left(\frac{4}{3} b_{2}-\frac{1}{6} b_{5}-\frac{4}{3} b_{9}-\frac{1}{6} b_{10}\right) \dot{a}_{(t)} k \cdots \text {. }
\end{aligned}
$$

USTEDM having first-order accuracy means

$$
\lim _{\Delta t \rightarrow 0} \frac{\varepsilon_{u_{t+2 \Delta t}}}{\Delta t}=0
$$

Combining (12) and (14) leads to

$$
\begin{aligned}
& \lim _{\Delta t \rightarrow 0}\left(\frac{d_{0}}{\Delta t}+d_{1}+d_{2} \Delta t+d_{3} \Delta t^{2}+\ldots\right) \\
& =\lim _{\Delta t \rightarrow 0}\left(\frac{\left(m+b_{1} c \Delta t+b_{2} k \Delta t^{2}\right) \varepsilon_{u_{t+2 \Delta t}}}{\Delta t}\right) \\
& =m \lim _{\Delta t \rightarrow 0}\left(\frac{\varepsilon_{u_{t+2 \Delta t}}}{\Delta t}\right)=0 .
\end{aligned}
$$

Equation (15) is satisfied only when

$$
d_{0}=d_{1}=0
$$

Combining (16) and (13) yields

$$
\begin{array}{r}
1-b_{3}-b_{6}=0, \\
2-b_{3}=0, \\
b_{1}-b_{4}-b_{7}=0 .
\end{array}
$$

Similarly, (8) has second-order accuracy only when $d_{0}=$ $d_{1}=d_{2}=0$. This means that

$$
\begin{aligned}
1-b_{3}-b_{6} & =0, \\
2-b_{3} & =0, \\
b_{1}-b_{4}-b_{7} & =0, \\
2-0.5 b_{3}-b_{10}-b_{9}-b_{11} & =0, \\
2 b_{1}-b_{4}-b_{9}-b_{10}-b_{11} & =0, \\
-b_{9}+b_{2}-b_{8}-b_{11}-b_{5}-b_{10} & =0
\end{aligned}
$$

Solving (18) yields

$$
\begin{aligned}
& b_{1}=1-b_{7}, \\
& b_{2}=1+b_{8}+b_{5}, \\
& b_{3}=2, \\
& b_{4}=1-2 b_{7}, \\
& b_{6}=-1, \\
& b_{11}=1-b_{10}-b_{9} .
\end{aligned}
$$

This shows that USTEDM is second order accurate. 
Similarly, (8) has third-order accuracy only when $d_{0}=$ $d_{1}=d_{2}=0$. This means that

$$
\begin{aligned}
& b_{1}=0.5, \\
& b_{2}=-b_{8}, \\
& b_{3}=2, \\
& b_{4}=0, \\
& b_{5}=-2 b_{8}-1, \\
& b_{6}=-1, \\
& b_{7}=0.5, \\
& b_{9}=b_{11}, \\
& b_{10}=-2 b_{11}+1, \\
& b_{11}=b_{11} .
\end{aligned}
$$

Equation (8) has fourth-order accuracy when $d_{0}=d_{1}=$ $d_{2}=d_{3}=d_{4}=0$, which can be satisfied when

$$
\begin{aligned}
b_{1} & =0.5, \\
b_{2} & =-b_{8}, \\
b_{3} & =2, \\
b_{4} & =0, \\
b_{5} & =-2 b_{8}-1, \\
b_{6} & =-1, \\
b_{7} & =0.5 \\
b_{9} & =b_{11}, \\
b_{10} & =-2 b_{11}+1, \\
\frac{1}{24} b_{3}-2 b_{9}-\frac{1}{2} b_{10}+\frac{2}{3} & =0 \\
\frac{4}{3} b_{1}-\frac{1}{6} b_{4}-2 b_{9}-\frac{1}{2} b_{10} & =0 \\
2 b_{2}-\frac{1}{2} b_{5}-2 b_{9}-\frac{1}{2} b_{10} & =0
\end{aligned}
$$

Equations (21) are contradictory equations, which shows that two-step displacement method expressed by (8) can have third-order accuracy at most, and (20) are adopted in the following derivation.

3.2. Stability Analysis. According to Lax theorem, (8) is convergent if and only if it is stable. The stability of (8) can be analyzed through studying a single degree-of-freedom (SDOF) system. For a SDOF system, (8) can be rewritten as

$$
\begin{aligned}
& \left\{\begin{array}{l}
u_{t+2 \Delta t} \\
u_{t+\Delta t}
\end{array}\right\} \\
& =\left[\begin{array}{cc}
\frac{b_{3} m+b_{4} c \Delta t+b_{5} k \Delta t^{2}}{m+b_{1} c \Delta t+b_{2} k \Delta t^{2}} & \frac{b_{6} m+b_{7} c \Delta t+b_{8} k \Delta t^{2}}{m+b_{1} c \Delta t+b_{2} k \Delta t^{2}} \\
1
\end{array}\right]\left\{\begin{array}{c}
u_{t+\Delta t} \\
u_{t}
\end{array}\right\} \\
& +\left\{\begin{array}{c}
\frac{b_{9} f_{t+2 \Delta t} \Delta t^{2}+b_{10} f_{t+\Delta t} \Delta t^{2}+b_{11} f_{t+\Delta t} \Delta t^{2}}{m+b_{1} c \Delta t+b_{2} k \Delta t^{2}} \\
0
\end{array}\right\} .
\end{aligned}
$$
is

Amplification matrix $A$ in (22) determining (8)'s stability

$$
\begin{aligned}
A & =\left[\begin{array}{ll}
A_{11} & A_{12} \\
A_{21} & A_{22}
\end{array}\right] \\
& =\left[\begin{array}{cc}
\frac{b_{3} m+b_{4} c \Delta t+b_{5} k \Delta t^{2}}{m+b_{1} c \Delta t+b_{2} k \Delta t^{2}} & \frac{b_{6} m+b_{7} c \Delta t+b_{8} k \Delta t^{2}}{m+b_{1} c \Delta t+b_{2} k \Delta t^{2}} \\
1 & 0
\end{array}\right] .
\end{aligned}
$$

Substituting $c=2 \xi \omega m, k=m \omega^{2}, \omega \Delta t=\Omega(\xi$-damp ratio, $\omega$-natural frequency) into (23) yields

$$
\begin{aligned}
A & =\left[\begin{array}{ll}
A_{11} & A_{12} \\
A_{21} & A_{22}
\end{array}\right] \\
& =\left[\begin{array}{cc}
\frac{b_{3}+2 b_{4} \xi \Omega+b_{5} \Omega^{2}}{1+2 b_{1} \xi \Omega+b_{2} \Omega^{2}} & \frac{b_{6}+2 b_{7} \xi \Omega+b_{8} \Omega^{2}}{1+2 b_{1} \xi \Omega+b_{2} \Omega^{2}} \\
1 & 0
\end{array}\right] .
\end{aligned}
$$

The characteristic equation of matrix $A$ can be written as

$$
\operatorname{det}(A-\lambda I)=\lambda^{2}-2 A_{1} \lambda+A_{2}=0,
$$

where $I$ is the identity matrix, $\lambda$ denotes eigenvalue of $A$, and

$$
\begin{aligned}
& A_{1}=\frac{1}{2} \operatorname{tr}(A)=\frac{A_{11}+A_{22}}{2}=\frac{1}{2} \frac{b_{3}+2 b_{4} \xi \Omega+b_{5} \Omega^{2}}{1+2 b_{1} \xi \Omega+b_{2} \Omega^{2}}, \\
& A_{2}=|A|=-\frac{b_{6}+2 b_{7} \xi \Omega+b_{8} \Omega^{2}}{1+2 b_{1} \xi \Omega+b_{2} \Omega^{2}} .
\end{aligned}
$$

The stability of (8) depends on spectral radius $\rho(A)$ of matrix $A$ and (8) is stable only when

$$
\rho(A) \leqslant 1 \text {. }
$$

Because $\rho(A)$ is determined by $A_{1}, A_{2}$, the stability domain of (8) can be expressed by functions $A_{1}$ and $A_{2}$. In similar way, the study of stability made by Hilber and Hughes [20] is performed. Firstly, the boundary line of stability domain of (8) where $\rho(A)=1$ is to be derived. $\rho(A)=1$ means that

$$
|\lambda|_{\max }=1
$$

where $|\lambda|_{\max }$ is the maximum amplitude of $\lambda$. Complex value $\lambda$ whose amplitude is 1 can be expressed by $\lambda=e^{i \varphi}$, where 
$i=\sqrt{-1}$ and $\varphi$ is argument of $\lambda$. Substituting $\lambda=e^{i \varphi}$ into (25) yields

$$
e^{i 2 \varphi}-2 A_{1} e^{i \varphi}+A_{2}=0 .
$$

The identities including $e^{i \varphi}=\cos \varphi+i \sin \varphi, \cos 2 \varphi=$ $2 \cos ^{2} \varphi-1, \sin 2 \varphi=2 \sin \varphi \cos \varphi$ are used and (29) can be rewritten as

$$
\begin{aligned}
& \left(2 \cos \varphi\left(\cos \varphi-A_{1}\right)+A_{2}-1\right) \\
& \quad+i\left(2 \sin \varphi\left(\cos \varphi-A_{1}\right)\right)=0 .
\end{aligned}
$$

Equation (30) is satisfied only when

$$
\begin{aligned}
2 \cos \varphi\left(\cos \varphi-A_{1}\right)+A_{2}-1 & =0, \\
2 \sin \varphi\left(\cos \varphi-A_{1}\right) & =0 .
\end{aligned}
$$

The boundary lines of stability domain of (8) can be determined by (31) when $\varphi \in[0,2 \pi]$. It can be derived that when $0<\varphi<\pi, \pi<\varphi<2 \pi$, (31) are satisfied when

$$
\begin{aligned}
& A_{1}=\cos \varphi, \\
& A_{2}=1 .
\end{aligned}
$$

When $\varphi=0, \varphi=2 \pi$, (31) are satisfied when

$$
1-2 A_{1}+A_{2}=0 .
$$

When $\varphi=\pi$, (31) are satisfied when

$$
1+2 A_{1}+A_{2}=0 .
$$

The boundary of stability domain of (8) is then made up with three lines described by (32), (33), and (34), respectively, in $A_{1}-A_{2}$ plane as shown in Figure 1. Because $\rho(A)$ is continuous function of $A_{1} A_{2}$ and when $A_{1}=A_{2}=$ $0, \rho(A)=0<1$, the stability domain of $(8)$ is the inner part of the triangle as shown in Figure 1 and can be expressed by

$$
\begin{aligned}
-1 & \leq A_{2} \leq 1, \\
-\frac{1}{2}\left(1+A_{2}\right) & \leq A_{1} \leq \frac{1}{2}\left(1+A_{2}\right) .
\end{aligned}
$$

3.3. Final Form of Two-Step Displacement Method with ThirdOrder Accuracy. Combining (20) and (8) yields

$$
\begin{aligned}
(m+ & \left.0.5 c \Delta t-b_{8} k \Delta t^{2}\right) u_{t+2 \Delta t} \\
= & \left(2 m+\left(-2 b_{8}-1\right) k \Delta t^{2}\right) u_{t+\Delta t} \\
& +\left(-1 m+0.5 c \Delta t+b_{8} k \Delta t^{2}\right) u_{t}+b_{11} f_{t+2 \Delta t} \Delta t^{2} \\
& +\left(-2 b_{11}+1\right) f_{t+\Delta t} \Delta t^{2}+b_{11} f_{(t)} \Delta t^{2} .
\end{aligned}
$$

Combining (20), (26), and (35) yields, after simplification, the stability domain of (36):

$$
\begin{aligned}
1-b_{8} \Omega^{2} & \geqslant 0, \\
1+\xi \Omega-b_{8} \Omega^{2} & >0, \\
4+\left(-4 b_{8}-1\right) \Omega^{2} & \geqslant 0 .
\end{aligned}
$$

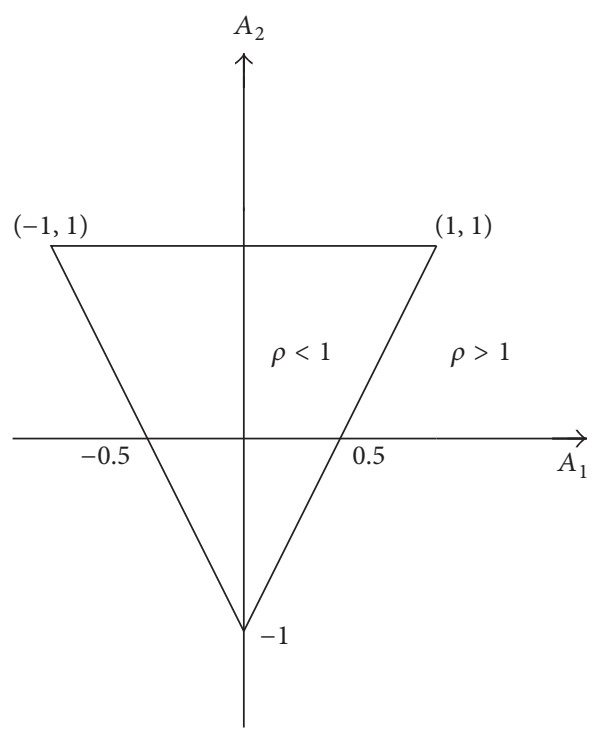

FIgURE 1: Stability domain of (8).

According to (36) and (37), the two-step displacement methods satisfying different requirements can be obtained. The explicitness of method can be obtained only when $b_{11}=0$ and the unconditional stability can be obtained when (37) are always satisfied when $\Omega \in[0, \infty)$. It can be found that (37) are always satisfied only when

$$
b_{8} \leqslant-0.25 \text {. }
$$

Substituting $b_{8}=-0.25, b_{11}=0$ into (36) yields

$$
\begin{aligned}
(m+ & \left.0.5 c \Delta t+0.25 k \Delta t^{2}\right) u_{t+2 \Delta t} \\
= & \left(2 m-0.5 k \Delta t^{2}\right) u_{t+\Delta t} \\
& +\left(-m+0.5 c \Delta t-0.25 k \Delta t^{2}\right) u_{t}+f_{t+\Delta t} \Delta t^{2} .
\end{aligned}
$$

Equation (39) shows the final form of USTEDM, which is an unconditionally stable two-step explicit integration algorithm. USTEDM is third order accurate. The analysis shows that USTEDM has very similar numerical attributes as Newmark's average acceleration method. Figure 2 shows that the spectral radius of amplification matrix of USTEDM is 1, which is the same as that of Newmark's average acceleration method.

Figure 3 shows that the numerical damping of USTEDM is zero, which is the same as that of Newmark's average acceleration method. Figure 4 shows the relative period error of USTEDM is minimal compared with other methods including Houbolt Method, Wilson- $\theta$ Method $(\theta=1.4)$, and Generalized $\alpha$ Method $\left(\alpha_{m}=0.3, \alpha_{f}=0.35\right)$. Though with similar numerical attributes as Newmark's average acceleration method, USTEDM is explicit and has great computation advantage over the implicit Newmark's average acceleration method.

The applicability of the USTEDM for nonlinear problem is also investigated. Compared with other unconditional 


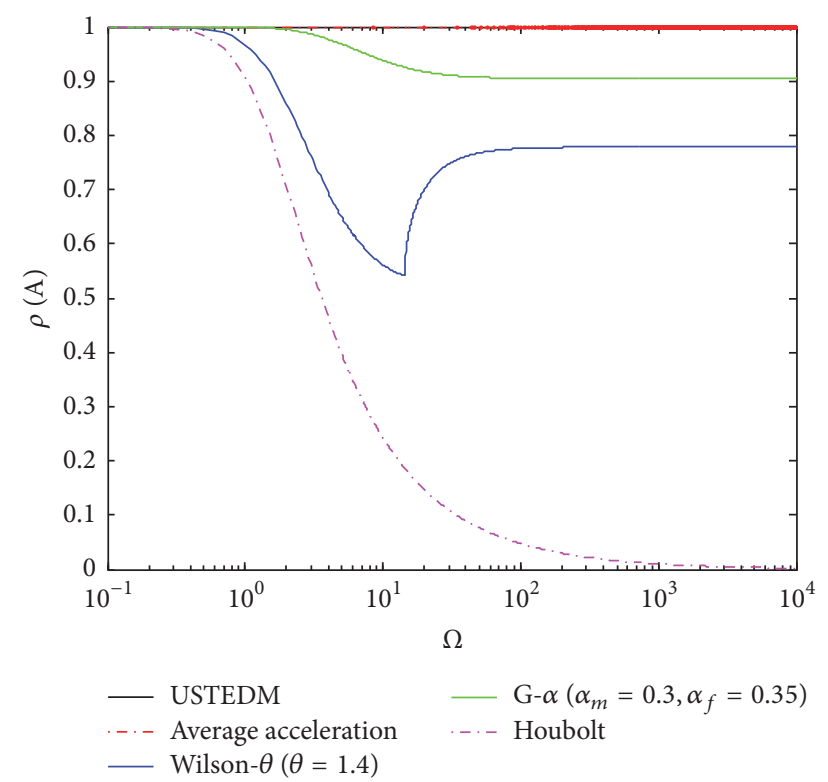

FIgURE 2: Comparison of spectral radius.

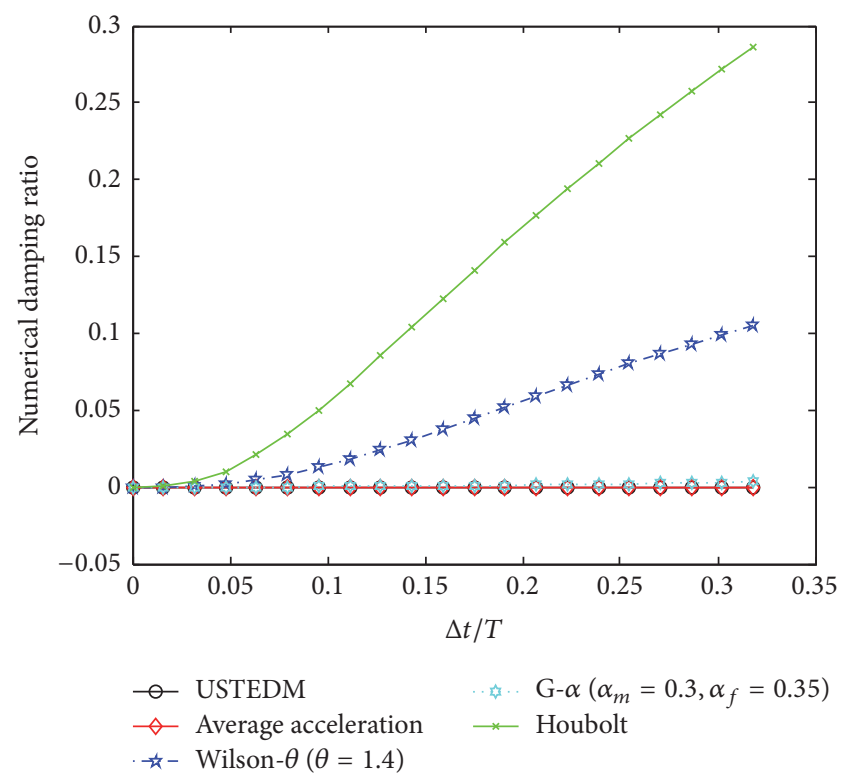

FIgURE 3: Comparison of numerical damping ratio.

stable explicit algorithms [17-19], whose main calculation amount includes at least solving two-reverse matrix of nondiagonal matrix for displacement and velocity, respectively, USTEDM shows higher calculation efficiency since its main calculation amount includes solving only onereverse matrix of nondiagonal matrix for displacement. In addition, USTEDM shows minimal memory requirements, since its calculation only involves displacement when only displacement-related nonlinearity occurs. This shows that USTEDM can be viewed as a complementary or improved form of conditional stable explicit CDM.

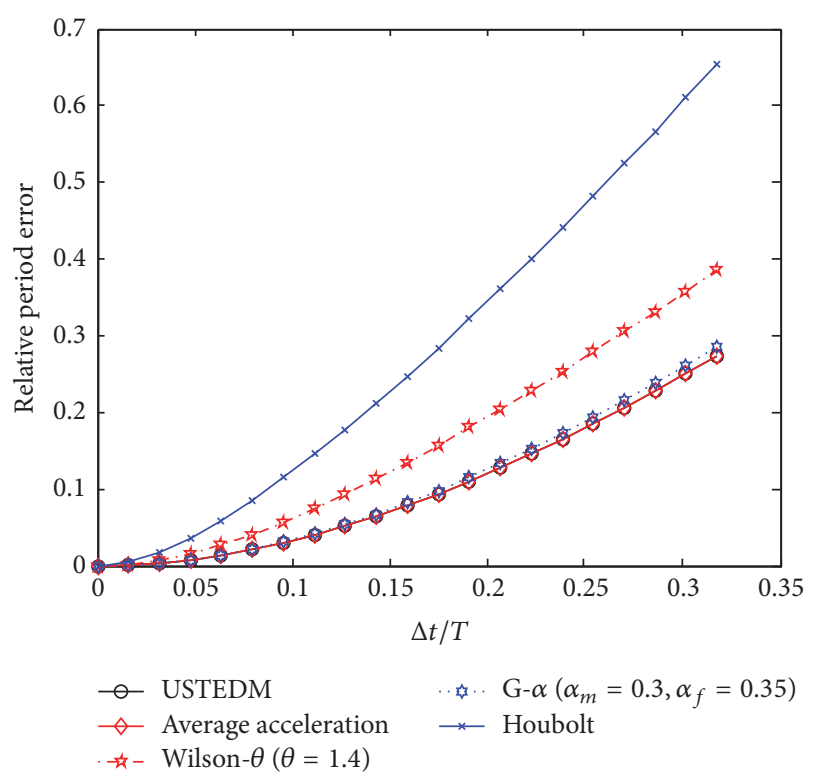

FIGURE 4: Comparison of relative period error.

3.4. Implementing USTEDM for Nonlinear Problem. The procedure to use USTEDM is similar to that of CDM and is outlined as follows.

(A) Initial input and calculation:

(1) Input the initial stiffness matrix $K_{0}$, mass matrix $M$, and damping matrix $C$.

(2) Input initial displacement vector $\left\{u_{0}\right\}$ and initial velocity vector $\left\{v_{0}\right\}$, solving initial acceleration vector $\left\{a_{0}\right\}:\left\{a_{0}\right\}=M^{-1}\left(\left\{f_{0}\right\}-K_{0}\left\{u_{0}\right\}-C\left\{v_{0}\right\}\right)$.

(3) Select time step duration $\Delta t$.

(4) Calculate

$$
\left\{u_{-\Delta t}\right\}=\left\{u_{0}\right\}-\left\{v_{0}\right\} \Delta t+\frac{1}{2}\left\{a_{0}\right\} \Delta t^{2} .
$$

(B) For each time step (from time point $t$ to time point $t+\Delta t)$

(1) based on known $\left\{u_{t}\right\},\left\{u_{t-\Delta t}\right\}, K_{t}$, and outer force vector $\left\{f_{t}\right\}$, calculate the "effective" load vector $\left\{\bar{f}_{t+\Delta t}\right\}$;

$$
\begin{aligned}
\left\{\bar{f}_{t+\Delta t}\right\}= & \left(2 M-0.5 K_{t} \Delta t^{2}\right)\left\{u_{t}\right\} \\
& +\left(-M+0.5 C \Delta t-0.25 K_{t} \Delta t^{2}\right)\left\{u_{t-\Delta t}\right\} \\
& +\left\{f_{t}\right\} \Delta t^{2}
\end{aligned}
$$

(2) form "effective" mass matrix: $\widehat{M}=M+0.5 C \Delta t+$ $0.25 K_{t} \Delta t^{2}$

(3) calculate $\left\{u_{t+\Delta t}\right\}=\widehat{M}^{-1}\left\{\widehat{f}_{t+\Delta t}\right\}$; 


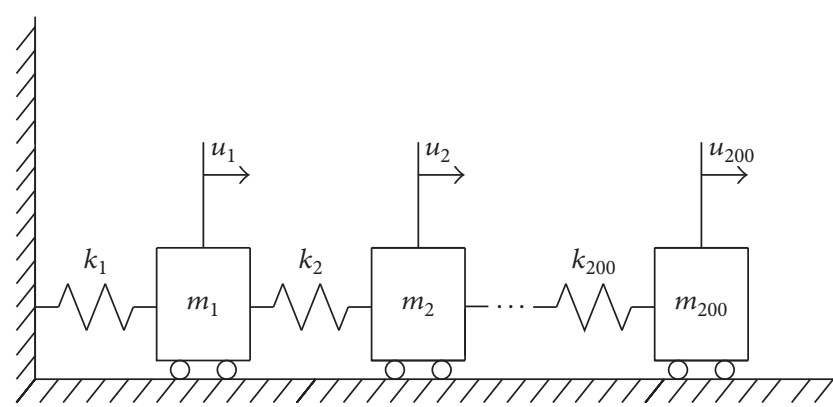

Figure 5: 200-DOF spring-mass system.

TABLE 1: Total time consumption (sec).

\begin{tabular}{lcc}
\hline USTEDM & Houbolt & Average acceleration \\
\hline 0.4825 & 353.0519 & 292.1621 \\
\hline
\end{tabular}

(4) if necessary, calculate velocity vector and acceleration vector at time point $t+\Delta t$ :

$$
\begin{gathered}
\left\{v_{t+\Delta t}\right\}=\frac{3\left\{u_{t+\Delta t}\right\}-4\left\{u_{t}\right\}+\left\{u_{t-\Delta t}\right\}}{2 \Delta t}, \\
\left\{a_{t+\Delta t}\right\}=M^{-1}\left(f_{t+\Delta t}-K_{t+\Delta t}\left\{u_{t+\Delta t}\right\}-C\left\{v_{t+\Delta t}\right\}\right) .
\end{gathered}
$$

3.5. Numerical Example. In this example [7, 17], the forced vibration response of an 200-degree-of-freedom spring-mass system as shown in Figure 5 is examined using the proposed numerical method. The structural properties of this system are assumed to be $m_{i}=100 \mathrm{~kg}$ and $k_{i}=10^{7}\left[1-\left(u_{i}-\right.\right.$ $\left.\left.u_{i-1}\right)^{2}\right] \mathrm{N} / \mathrm{m}$ in which $i=1,2, \ldots, 200$. This system is subjected to a ground acceleration of $10 \sin (\pi t)$. The lowest and highest natural frequency is $2.62 \mathrm{rad} / \mathrm{s}$ and $632.4 \mathrm{rad} / \mathrm{s}$, respectively [17]. According to the highest natural frequency, a time step duration of $\Delta t=0.001 \mathrm{~s}$ is obtained from the Newmark's average acceleration method and is selected to be the exact solution. Displacement responses versus time of the largest degree of freedom are plotted in Figure 6. Analysis is accomplished by considering time step duration to be equal to $0.02 \mathrm{~s}$. The displacement errors are presented in Figure 7. It can be seen that here USTEDM provides the most accurate displacement solutions, compared with Houbolt Method and commonly used Newmark's average acceleration method. More importantly, without needs of iteration calculation, the unconditionally stable explicit USTEDM has much less time consumption of calculation, compared with those unconditionally stable implicit methods, which are listed in Table 1.

\section{Conclusions}

A novel two-step unconditionally stable explicit displacement method, named Unconditional Stable Two-Step Explicit Displacement Method (USTEDM), is proposed in this paper. USTEDM has third-order accuracy and is unconditionally stable. The conclusions mainly include the following:

(1) The novel way of constructing direct time integration methods from the start point of dimensional analysis

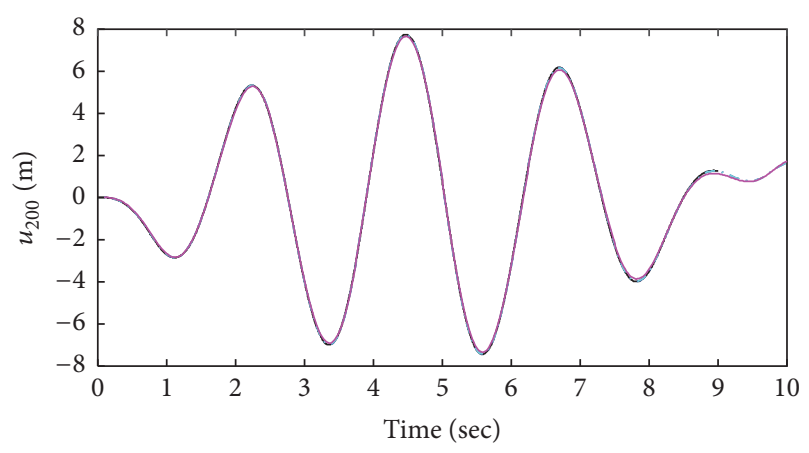

$$
\begin{aligned}
& \text { _- Exact solution } \\
& - \text {-.- USTEDM } \\
& \ldots-\text { Newmark average acceleration method } \\
& \text { - Houbolt method }
\end{aligned}
$$

Figure 6: Displacement responses of 200th DOF system.

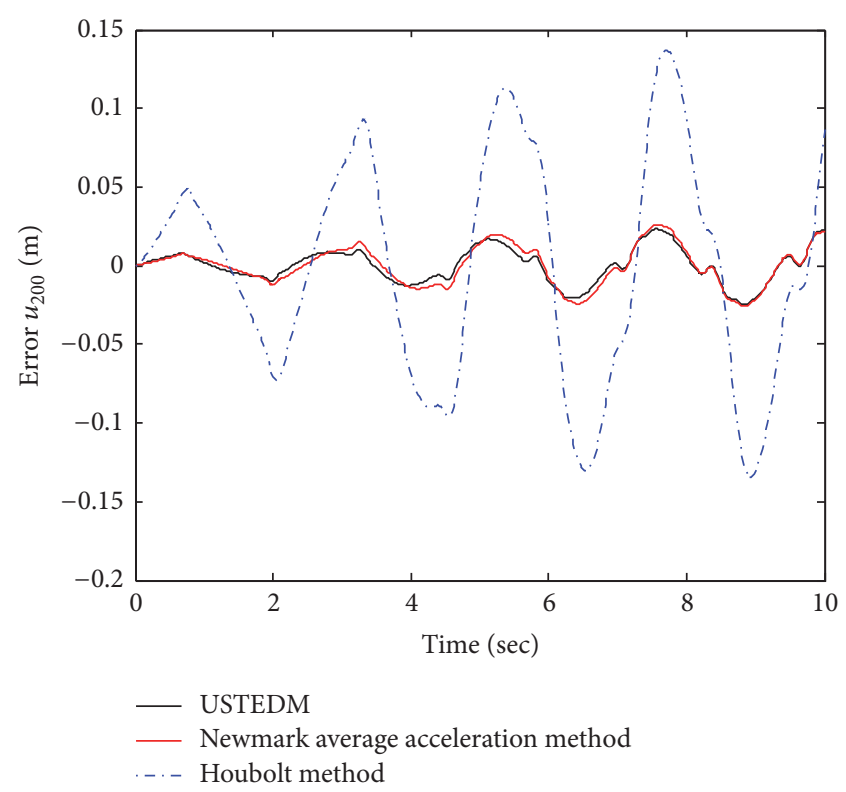

FIGURE 7: Comparison of displacement errors of 200-DOF system.

is applicable, and it is believed that it will contribute to improvement of numerical calculation in structural dynamics.

(2) With no prerequisite difference assumption of velocity and acceleration, the derivation of USTEDM is completely different from any documented one. With unconditional stability, third-order accuracy, higher calculation efficiency, and lower storage requirement, USTEDM is promising in solving vibrations of structures, especially those with displacement-dependent nonlinearity.

The zero numerical damping of USTEDM is its shortcoming, and the introduction of numerical damping will be studied. 


\section{Competing Interests}

The authors declare that they have no competing interests.

\section{Acknowledgments}

The authors would like to acknowledge the partial support of this work from Project Research on Parallel Adaptive Method in Solving Seismic Response of High Speed Railway Bridge supported by National Natural Science Foundation of China (51308555) and Hunan Provincial Natural Science Foundation of China (14JJ3015). The research was supported by the Postdoctoral Science Foundation of Central South University and the Strategic Pilot Project of Central South University.

\section{References}

[1] J. C. Houbolt, "A recurrence matrix solution for the dynamic response of aircraft in gusts," Journal of the Aeronautical Sciences, vol. 17, no. 9, pp. 540-550, 1950.

[2] N. M. Newmark, "A method for computation of structural dynamics," Journal of Engineering Mechanics (ASCE), vol. 85, pp. 67-94, 1959.

[3] E. L. Wilson, I. Farhoomand, and K. J. Bathe, "Nonlinear dynamic analysis of complex structures," Earthquake Engineering and Structural Dynamics, vol. 1, no. 3, pp. 241-252, 1973.

[4] T. C. Fung, "Third order complex-time-step methods for transient analysis," Computer Methods in Applied Mechanics and Engineering, vol. 190, no. 22-23, pp. 2789-2802, 2001.

[5] K.-J. Bathe, "Conserving energy and momentum in nonlinear dynamics: a simple implicit time integration scheme," Computers and Structures, vol. 85, no. 7-8, pp. 437-445, 2007.

[6] K.-J. Bathe and G. Noh, "Insight into an implicit time integration scheme for structural dynamics," Computers and Structures, vol. 98-99, pp. 1-6, 2012.

[7] A. Akbar Gholampour, M. Ghassemieh, and M. Karimi-Rad, "A new unconditionally stable time integration method for analysis of nonlinear structural dynamics," Journal of Applied Mechanics, vol. 80, no. 2, pp. 111-121, 2013.

[8] W.-M. Zhai, "Two simple fast integration methods for largescale dynamic problems in engineering," International Journal for Numerical Methods in Engineering, vol. 39, no. 24, pp. 4199$4214,1996$.

[9] G. M. Hulbert and J. Chung, "Explicit time integration algorithms for structural dynamics with optimal numerical dissipation," Computer Methods in Applied Mechanics and Engineering, vol. 137, no. 2, pp. 175-188, 1996.

[10] S.-Y. Chang, "An explicit method with improved stability property," International Journal for Numerical Methods in Engineering, vol. 77, no. 8, pp. 1100-1120, 2009.

[11] G. Noh and K.-J. Bathe, "An explicit time integration scheme for the analysis of wave propagations," Computers and Structures, vol. 129, pp. 178-193, 2013.

[12] C. Yang, S. Xiao, L. Lu, and T. Zhu, "Two dynamic explicit methods based on double time steps," Proceedings of the Institution of Mechanical Engineers, Part K: Journal of Multi-body Dynamics, vol. 228, no. 3, pp. 330-337, 2014.

[13] C. Li and L. Jiang, "Explicit concomitance of implicit method to solve vibration equation," Journal of Earthquake Engineering and Engineering Vibration, vol. 11, no. 2, pp. 269-272, 2012.
[14] J. Har and K. K. Tamma, Computational Dynamics of Particles, Materials and Structures, John Wiley \& Sons, 2012.

[15] S. U. Masuri, A. Hoitink, X. Zhou, and K. K. Tamma, "Algorithms by design: a new normalized time-weighted residual methodology and design of a family of energy-momentum conserving algorithms for non-linear structural dynamics," International Journal for Numerical Methods in Engineering, vol. 79, no. 9, pp. 1094-1146, 2009.

[16] K. K. Tamma, J. Har, X. Zhou, M. Shimada, and A. Hoitink, "An overview and recent advances in vector and scalar formalisms: space/time discretizations in computational dynamics-a unified approach," Archives of Computational Methods in Engineering, vol. 18, no. 2, pp. 119-283, 2011.

[17] S.-Y. Chang, "Improved explicit method for structural dynamics," Journal of Engineering Mechanics, vol. 133, no. 7, pp. 748760, 2007.

[18] C. Kolay and J. M. Ricles, "Development of a family of unconditionally stable explicit direct integration algorithms with controllable numerical energy dissipation," Earthquake Engineering and Structural Dynamics, vol. 43, no. 9, pp. 1361-1380, 2014.

[19] C. Kolay and J. M. Ricles, "Assessment of explicit and semiexplicit classes of model-based algorithms for direct integration in structural dynamics," International Journal for Numerical Methods in Engineering, vol. 107, no. 1, pp. 49-73, 2016.

[20] H. M. Hilber and T. J. Hughes, "Collocation, dissipation and [overshoot] for time integration schemes in structural dynamics," Earthquake Engineering \& Structural Dynamics, vol. 6, no. 1, pp. 99-117, 1978. 


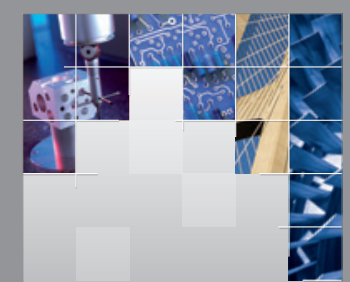

\section{Enfincering}
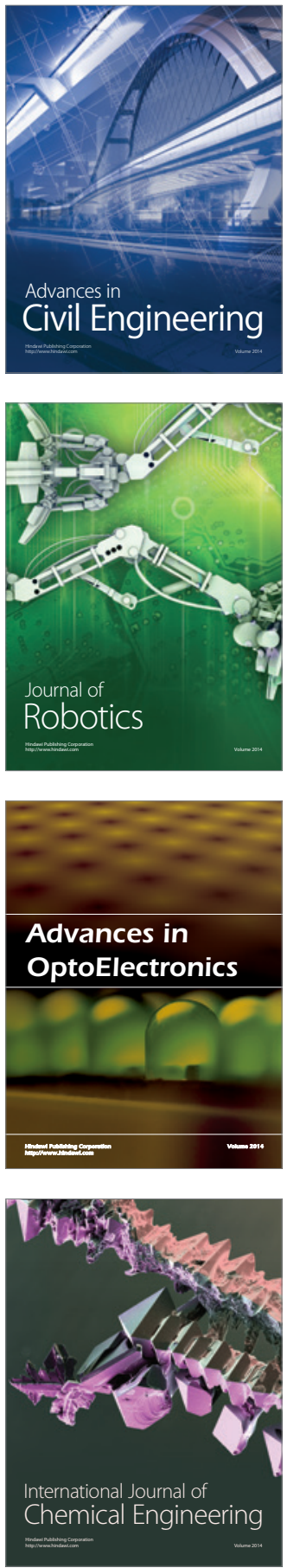

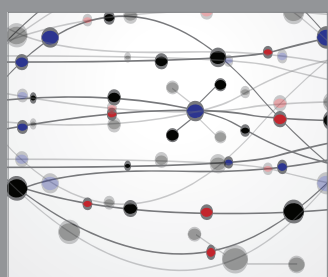

The Scientific World Journal

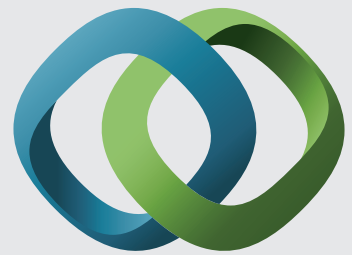

\section{Hindawi}

Submit your manuscripts at

http://www.hindawi.com
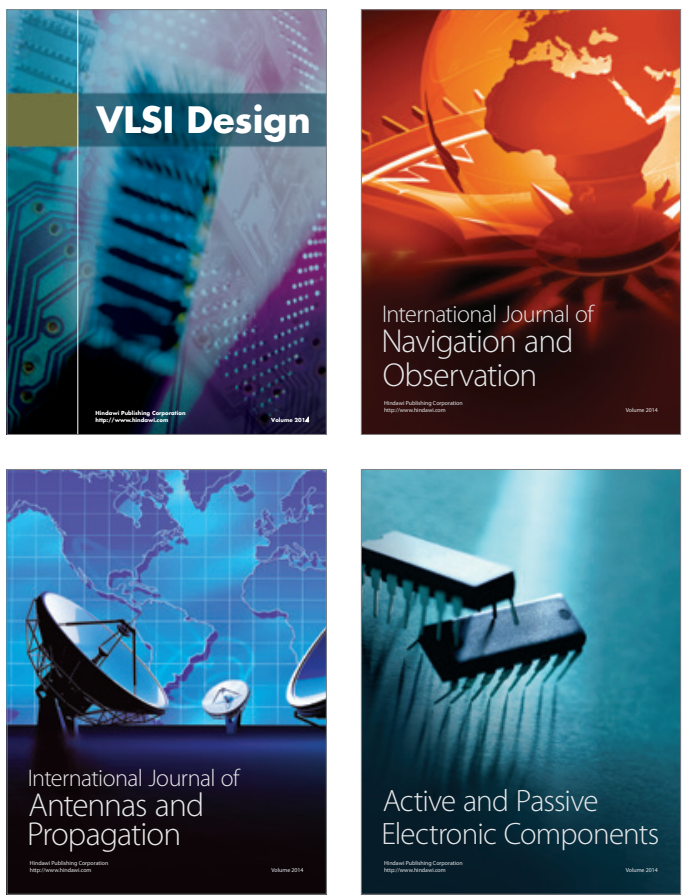
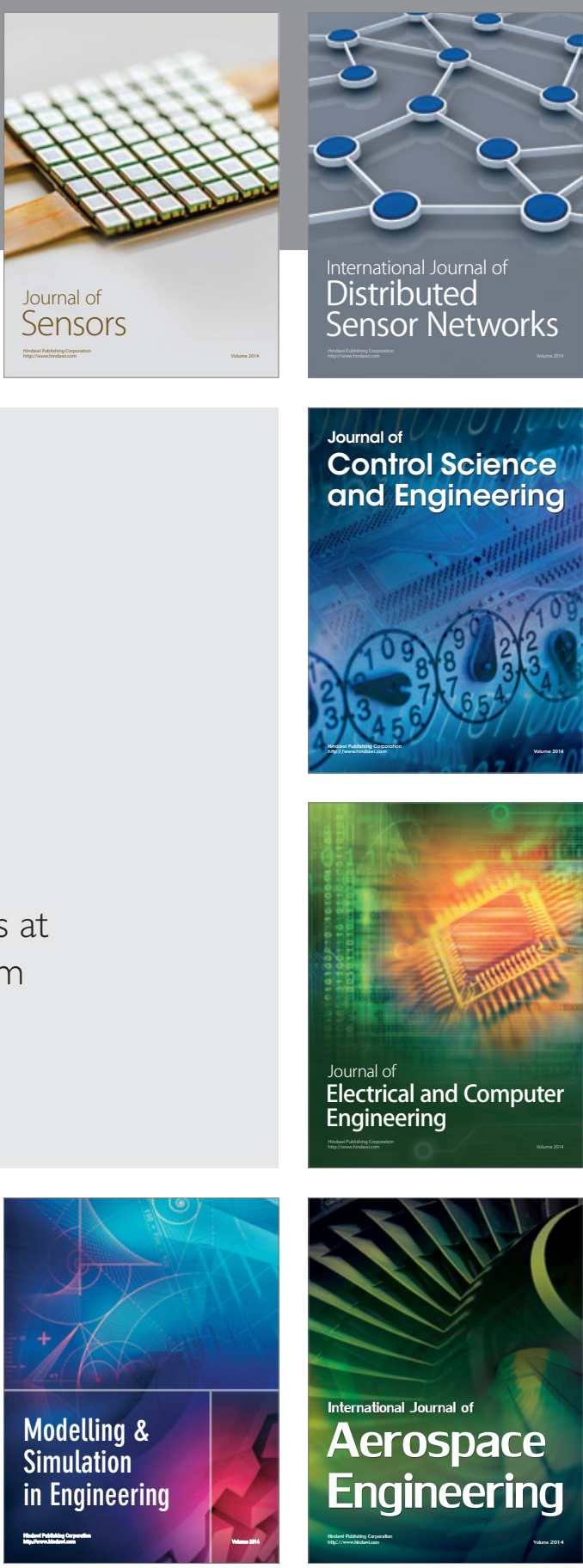

International Journal of

Distributed

Sensor Networks

Journal of

Control Science

and Engineering
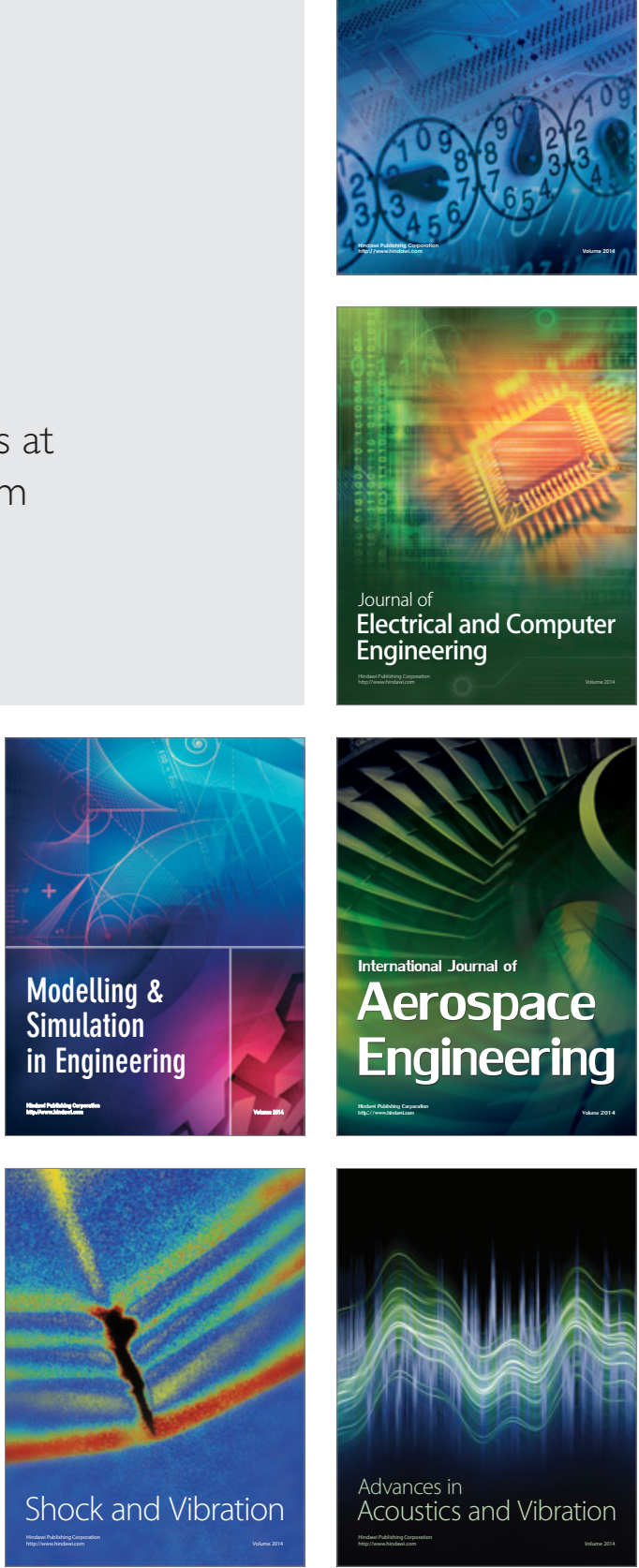